\title{
Fusarium solani
}

National Cancer Institute

\section{Source}

National Cancer Institute. Fusarium solani. NCI Thesaurus. Code C128551.

A species of non-dermatophytic hyaline mold in the phylum Ascomycota. The species is a ubiquitous soil borne pathogen causing several diseases on a broad range of plants. 